\title{
Third Time's a Charm: Immunization Strategies for Hepatitis B in Immunosuppressed Patients with Inflammatory Bowel Disease
}

\author{
Dana Ley $^{1} \cdot$ Mary S. Hayney ${ }^{2} \cdot$ Freddy Caldera $^{3}$ \\ Published online: 11 May 2019 \\ (c) Springer Science+Business Media, LLC, part of Springer Nature 2019
}

The clinical spectrum of hepatitis B (HepB) virus infection ranges from asymptomatic infection to fulminant hepatitis. Since both mild and fulminant hepatitis B infections have been reported in previously vaccinated patients with inflammatory bowel disease (IBD) receiving immunosuppressive therapies [1], current gastroenterology preventive care guidelines recommend HepB vaccination for all patients with IBD [2]. Indeed, patients receiving tumor necrosis factor (TNF) blocking antibodies were less likely to have protective hepatitis B surface antibody (HBsAb) levels following HepB vaccination [3]. Current evidence points to lifelong protection with $\mathrm{HBsAb}>10 \mathrm{mIU} / \mathrm{mL}$ measured 1-2 months after HepB vaccine series completion in immunocompetent adults; revaccination may be needed for individuals who fail to achieve that goal [4]. Previously immunized individuals may not have had an antibody level measured upon completion of the series. A single dose of HepB vaccine followed by HBsAb measurement in 1 month is used to document an anamnestic response and long-term immunity. $\mathrm{HBsAb}<10 \mathrm{mIU} / \mathrm{mL}$ after a single dose is considered an absence of an anamnestic response, requiring two more doses to complete a second series. Little work has been done to consider the optimal revaccination strategy in immunosuppressed patients with IBD, including how many additional doses of $\mathrm{HepB}$ vaccine are needed.

In this issue of Digestive Diseases and Sciences, Pratt and colleagues address this paucity of data addressing

Freddy Caldera

fcaldera@medicine.wisc.edu

1 Division of Internal Medicine, Department of Medicine, School of Medicine and Public Health, University of Wisconsin - Madison, Madison, USA

2 School of Pharmacy, School of Medicine and Public Health, University of Wisconsin - Madison, Madison, USA

3 Division of Gastroenterology and Hepatology, Department of Medicine, School of Medicine and Public Health, University of Wisconsin-Madison, 1685 Highland Avenue, Madison, WI 53705-2281, USA
HepB revaccination strategies [5]. The authors performed a retrospective cohort study at a single academic center that included 149 patients with IBD who failed to mount an immune response after a HepB vaccine series, which they referred to as primary vaccination failure. They compared $\leq 2$ versus 3 revaccination doses, finding a statistically significant positive association between the number of revaccination doses and achieving vaccine-induced immunity (HBsAb > 10). In their multivariable model, these results were statistically significant for patients of all ages.

This study was novel in that it compared two HepB revaccination strategies within one cohort. Its findings showed that three revaccination doses were significantly more likely to induce an immune response than one or two revaccination doses. Secondary analysis stratified patients by age less than or greater than 40 years. Those over age 40 years also responded significantly more frequently to revaccination with three doses, although less well compared to those aged $<40$ years of age. Pratt and colleagues ultimately conclude that gastroenterologists should follow the Advisory Committee on Immunization Practice (ACIP) recommendation to check HBsAb levels 1-2 months after HepB vaccine series in immunosuppressed patients with IBD [4]. They recommend that if a patient's antibody concentration is $<10 \mathrm{mIU} / \mathrm{mL}$, a full three dose series should be repeated at that time. These findings should help inform future guidelines on revaccination strategy in this patient group.

The main limitation of this study was that the authors could not differentiate between those who failed to mount a response to the vaccine series and those who only had waning HBsAb levels but were still immune. Although HBsAb levels are used to determine immunity and a value of $\geq 10$ $\mathrm{mIU} / \mathrm{mL}$ is a surrogate of clinical protection, HBsAb levels wane over time [4]. Even if the HBsAb is $<10 \mathrm{mIU} / \mathrm{mL}$, a person is considered to be immune if an anamnestic response is elicited as described above. The majority of individuals (88\%) who completed the HepB vaccine series as children or adults with an HBsAb level $<10$ at 30 years developed 
an antibody response of $\geq 10 \mathrm{mIU} / \mathrm{mL}$ after receiving a challenge dose of HepB vaccine, indicating persistent immunity [6]. Therefore, the HepB vaccine provides sustained protection in immunocompetent adults, but it is currently unknown if protection persists for immunosuppressed patients with IBD. Several immunosuppressive medications may not affect sustained immunity to HepB: Thiopurines have little effect on the sustained immunity to some vaccines, whereas as-stated anti-TNF therapy can blunt the response [7, 8]. It is unknown which immunosuppressive regimens may affect sustained immunity to hepatitis B.

The other significant limitation was that there were differences between the study arms. For example, there was a higher percentage of immunosuppressed patients within the $\leq 2$ dose arm compared to the three dose arm. Even though this study had limitations that influenced the conclusions that could be drawn, it provided important, novel findings that suggest that in patients with IBD who completed a HepB vaccine series and $\mathrm{HBsAb}<10 \mathrm{mIU} / \mathrm{mL}$, vaccine response is more likely following three additional HepB vaccination doses rather than 1-2 doses. These results can be generalized to patients of any age.

A recently licensed HepB vaccine, Heplisav-B ${ }^{\circledR}$ (HepB$\mathrm{CpG}$ ), may improve vaccine response rate in immunosuppressed patients with IBD. Similar to the other HepB vaccines, the antigen is based on recombinantly expressed purified hepatitis B surface antigen, but unlike conventional aluminum salt adjuvants, HepB-CpG contains an adjuvant consisting of a single-stranded cytosine-phosphodiester-guanosine (CpG) oligodeoxynucleotide, a damage-associated molecular pattern that is a ligand for Toll-like receptor (TLR)-9, an immune receptor that when activated substantially amplifies the immune response to the vaccine antigen [9]. HepB-CpG is administered as a two-dose series with just 4 weeks between doses. The ACIP lists HepB-CpG as an option for prevention of hepatitis B infection in adults but did not make a recommendation for its use in any specific patient population [10]. HepB-CpG series was superior to a series using conventional aluminum adjuvant $\mathrm{HepB}$ vaccine in patients with type 2 diabetes, in healthy individuals aged 11-55 years, and in adults aged 40-70 years [10]. Note that individuals with underlying diseases, such as immunosuppressing conditions, type 2 diabetes, and older age are less likely to mount a protective immune response to a series with conventional (aluminum adjuvant) HepB vaccine [4]. Although no data exist regarding the effectiveness of HepB-CpG in immunosuppressed populations such as patients with IBD, HepB-CpG offers the advantage of completion of the series in just 1 month. This vaccine preparation may be best suited for the first immunization series. It could also be used as the revaccination series in individuals who did not mount an anamnestic response to a HepB vaccine challenge dose as the series can be completed in just 4 weeks compared to 5 months required by a series with conventional (aluminum adjuvant) HepB vaccine.

In conclusion, immunosuppressed patients with IBD who received a HepB vaccine series, have $\mathrm{HBs} A b<10 \mathrm{mIU} / \mathrm{mL}$, and do not respond to a challenge dose of HepB vaccine should receive a total of three doses of vaccine. Future prospective or randomized controlled studies should be pursued to determine the prevalence of anamnestic responses and the true rate of nonresponse to the primary three dose series. Additionally, future studies could attempt to determine the risk of HepB vaccine nonresponse and the effect of immunosuppressive drugs for both primary series and revaccination when needed. Further work is needed to determine the optimal immunization strategy; prospective studies evaluating whether the new adjuvant HepB-CpG has superior rates of achieving immunity are needed.

\section{References}

1. Gisbert JP, Chaparro M, Esteve M. Review article: prevention and management of hepatitis B and C infection in patients with inflammatory bowel disease. Aliment Pharmacol Ther. 2011;33:619-633.

2. Farraye FA, Melmed GY, Lichtenstein GR, Kane SV. CG clinical guideline: preventive care in inflammatory bowel disease. Am J Gastroenterol. 2017;112:241-258.

3. Pratt PK Jr, Weber HC, David N, et al. Antibody response to hepatitis $B$ virus vaccine is impaired in patients with inflammatory bowel disease on infliximab therapy. Inflamm Bowel Dis. 2018;24:380-386.

4. Schillie S, Vellozzi C, Reingold A, et al. Prevention of hepatitis B virus infection in the United States: recommendations of the advisory committee on immunization practices. MMWR Recomm Rep. 2018;67:1-31.

5. Pratt PK, Nunes D, Long MT, Farraye FA. Improved antibody response to three additional hepatitis $\mathrm{B}$ vaccine doses following primary vaccination failure in patients with inflammatory bowel disease. Dig Dis Sci. (Epub ahead of print). https://doi.org/10.1007/ s10620-019-05595-6.

6. Zanis C, Bruden D, Hurlburt D, et al. Antibody levels and protection after hepatitis B vaccine: results of a 30-year follow-up study and response to a booster dose. J Infect Dis. 2016;214:16-22.

7. Caldera F, Saha S, Wald A, et al. Lower sustained diphtheria and pertussis antibody concentrations in inflammatory bowel disease patients. Dig Dis Sci. 2018;63:1532-1540.

8. Dotan I, Werner L, Vigodman S, et al. Normal response to vaccines in inflammatory bowel disease patients treated with thiopurines. Inflamm Bowel Dis. 2012;18:261-268.

9. Heplisav-B [Hepatitis B Vaccine (Recombinant), Adjuvanted] Package Insert Dynavax Technologies Corporation. 2018.

10. Schillie S, Harris A, Link-Gelles R, Romero J, Ward J, Nelson N. Recommendations of the advisory committee on immunization practices for use of a hepatitis B vaccine with a novel adjuvant. MMWR Morb Mortal Wkly Rep. 2018;67:455-458.

Publisher's Note Springer Nature remains neutral with regard to jurisdictional claims in published maps and institutional affiliations. 